\title{
ESTUDO CINÉTICO POR TG E DSC DA DECOMPOSIÇÃO TÉRMICA DE ALGUNS ADUTOS DE HALETOS DE CÁDMIO
}

Robson Fernandes de Farias

Departamento de Química - Universidade Federal de Roraima - Av. Venezuela s/n, 69310-270 - Boa Vista - RR

Ótom Anselmo de Oliveira, Hélio Scatena Júnior, Filipe Martel de Magalhães Borges e Ademir Oliveira da Silva

Departamento de Química - Universidade Federal do Rio Grande do Norte - Av. Senador Salgado Filho s/n - CP 1662 - 59072-970

- Natal - RN

\begin{abstract}
KINETICAL STUDY BY TGA AND DSC DATA OF THE THERMAL DECOMPOSITION OF SOME CADMIUM HALIDE ADDUCTS. By DSC data, the kinetical parameters $\mathrm{E}_{\mathrm{a}}$, $\mathrm{n}$ and $\mathrm{A}$ were calculated for the thermal decomposition of the adducts $\mathrm{CdCl}_{2} .2 \mathrm{dmf}, \mathrm{CdCl}_{2} . \mathrm{dmf}$ and $\mathrm{CdBr}_{2} . \mathrm{dmf}$ $(\mathrm{dmf}=$ dimethylformamide $)$ by using Rogers and Smith method. The found values were $: \mathbf{E}_{\mathrm{a}}=\mathbf{8 5}$, 176 and $101 \mathrm{~kJ} \mathrm{~mol}^{-1}, \mathrm{n}=0.23,0.25$, and $0.17, A=2.40 \times 10^{9}, 1.89 \times 10^{19}$ and $1.07 \times 10^{9}$ respectively. By TGA data, the kinetical patameters for the thermal decomposition of the adduct $\mathrm{CdCl}_{2.1,5}$ dmeu (dmeu=dimethylethyleneurea) were calculated by using five different methods.
\end{abstract}

Keywords : thermal decomposition; kinetical parameters; cadmium adducts.

\section{INTRODUÇÃO}

A dimetilformamida (dmf) é a mais simples das amidas $\mathrm{N}$ substituidas e, devido às suas características, é uma das amidas mais utilizadas como solvente em processos industriais e no laboratório ${ }^{1}$. A dimetiletilenouréia (dmeu), diferentemente da etilenouréia da qual é derivada, é liquída à temperatura ambiente, chegando a ser utilizada como solvente em sínteses ou estudos espectroscópicos ${ }^{2,3}$.

O estudo cinético da termodecomposição de sólidos pode ser efetuado utilizando-se dados de TG, DTA ou DSC ${ }^{4}$. Comumente, este tipo de estudo é aplicado à desidratação de sais, decomposição de polímeros, explosivos e compostos de coordenação.

Neste trabalho analisa-se, do ponto de vista cinético, a decomposição térmica dos adutos $\mathrm{CdCl}_{2}$. $2 \mathrm{dmf}, \mathrm{CdCl}_{2} \cdot \mathrm{dmf}$ e $\mathrm{CdBr}_{2} \cdot \mathrm{dmf}^{5,6}$, através de dados de DSC, utilizando-se o método de Rogers e Smith $^{7}$ e, através de dados de termogravimetria, estuda-se cineticamente a decomposição do aduto $\mathrm{CdCl}_{2} .1,5 \mathrm{dmeu}^{8}$ utilizando-se os métodos de Coats-Redfern ${ }^{9}$, Freeman ${ }^{10}$ Horovitz $^{11}$, Blazejowski ${ }^{12}$ e Zsakó ${ }^{13}$.

O principal objetivo do presente trabalho é correlacionar, de forma a mais precisa possível, os parâmetros cinéticos calculados $\left(\mathrm{E}_{\mathrm{a}}=\right.$ energia de ativação, $\mathrm{n}=$ ordem de reação e $\mathrm{A}=$ fator pré-exponencial) com as características estruturais dos compostos estudados, tentando-se dar a estes termos um significado físico adequado quando os aplicamos à termodecomposição de compostos de coordenação. São propostas hipóteses que, pretendemos, sejam objeto de estudo em trabalhos mais extensos e minuciosos a serem realizados posteriormente.

\section{PARTE EXPERIMENTAL}

\section{Síntese e Análise Elementar dos Compostos}

$\mathrm{O}$ aduto $\mathrm{CdCl}_{2} .2 \mathrm{dmf}$ foi sintetizado pela dissolução de $1,8 \mathrm{~g} \mathrm{de} \mathrm{CdCl}_{2}$ em $15 \mathrm{~cm}^{3}$ de dmf. A solução produzida foi colocada em banho de gelo e deixada em agitação por uma hora, sendo o excesso de ligante removido posteriormente por evaporação a vácuo, sendo que, durante a evaporação, o aduto forma aglomerados que dificultam a secagem, que se completa apenas após $20 \mathrm{~h}$.

$\mathrm{O} \mathrm{CdCl}_{2}$.dmf foi obtido pela dissolução de $1,8 \mathrm{~g}$ de $\mathrm{CdCl}_{2}$ em 1,2-dicloroetano sendo em seguida adicionados a esta solução $0,72 \mathrm{~g}$ de dmf, deixando-se a mistura resultante em agitação por quatro horas. Durante o período de agitação, houve a formação de precipitado que foi filtrado em placa porosa, lavado três vezes com éter etílico e seco a vácuo.

$\mathrm{O} \mathrm{CdBr}_{2} . \mathrm{dmf}$ foi preparado pela dissolução de $2,0 \mathrm{~g} \mathrm{de} \mathrm{CdBr}_{2}$ em $15 \mathrm{~cm}^{3}$ de dmf deixando-se a solução em agitação por duas horas. Em seguida, adicionou-se éter etílico à solução, o que promoveu a precipitação do aduto, que foi então filtrado em placa porosa e seco a vácuo.

$\mathrm{O} \mathrm{CdCl}_{2}$. 1,5 dmeu foi obtido misturando-se $1,5 \mathrm{~g} \mathrm{de} \mathrm{CdCl}_{2}$ a $15 \mathrm{~cm}^{3}$ de dmeu, deixando-se a mistura em agitação por $24 \mathrm{~h} . \mathrm{O}$ aduto formado foi lavado com éter etílico e seco a vácuo.

Para todos os adutos, a determinação dos teores de metal foi efetuada por titulação complexométrica com EDTA e a determinação de haletos, por titulação potenciométrica com $\mathrm{AgNO}_{3}{ }^{14}$.

Para os adutos com dmf, os teores de nitrogênio foram determinados pelo método de Kjeldahl ${ }^{14}$. Para o aduto com dmeu, os teores de $\mathrm{C}, \mathrm{N}$ e $\mathrm{O}$ foram determinados em microanalisador do IQ da USP.

\section{Aparelhagem}

As curvas DSC a partir das quais os parâmetros cinéticos foram calculados para os compostos com dmf, foram obtidas em um DSC da Perkin -Elmer, com uma taxa de aquecimento de $4,2 \times 10^{-2} \mathrm{~K} \mathrm{~s}^{-1}$. A curva TG para o $\mathrm{CdCl}_{2}$. $1,5 \mathrm{dmeu}$ foi obtida num $\mathrm{TGA}^{-7}$ da Perkin-Elmer em atmosfera de $\mathrm{N}_{2}$ à $0,16 \mathrm{~K} \mathrm{~s}^{-1}$.

\section{RESULTADOS E DISCUSSÃO}

1) $\mathrm{CdCl}_{2} .2 \mathrm{dmf}, \mathrm{CdCl}_{2} \cdot \mathrm{dmf}$ e $\mathrm{CdBr}_{2} \cdot \mathrm{dmf}$

Analisando-se as curvas termograviméticas obtidas, ${ }^{5,6}$, verificase que para os três compostos, a decomposição térmica inicia-se 
pela saída dos ligantes, ocorrendo em seguida a sublimação do haleto. Para o $\mathrm{CdCl}_{2} .2 \mathrm{dmf}$, a perda dos ligantes acontece em duas etapas, uma para cada ligante :

$$
\begin{gathered}
\mathrm{CdCl}_{2} \cdot 2 \mathrm{dmf}(\mathrm{s})=\mathrm{CdCl}_{2} \cdot \mathrm{dmf}^{*}(\mathrm{~s})+\mathrm{dmf}(\mathrm{g}) \\
\mathrm{CdCl}_{2} \cdot \mathrm{dmf}^{*}(\mathrm{~s})=\mathrm{CdCl}_{2}(\mathrm{~s})+\mathrm{dmf}(\mathrm{g})
\end{gathered}
$$

Os parâmetros cinéticos calculados são apresentados na tabela 1:

Tabela 1. Parâmetros cinéticos para alguns adutos entre dmf e haletos de Cd (método de Rogers e Smith)

\begin{tabular}{lcccc}
\hline composto & $\begin{array}{c}\mathrm{E}_{\mathrm{a}} \\
\left(\mathrm{kJmol}^{-1}\right)\end{array}$ & $\mathrm{n}$ & $\mathrm{A}$ & $\begin{array}{c}\mathrm{T}_{\mathrm{i}}-\mathrm{T}_{\mathrm{f}} \\
(\mathrm{K})\end{array}$ \\
\hline $\mathrm{CdCl}_{2} \cdot 2 \mathrm{dmf}$ & 85 & 0,23 & $2,40 \times 10^{9}$ & $339-378$ \\
$\mathrm{CdCl}_{2} \cdot \mathrm{dmf}$ & 176 & 0,25 & $1,89 \times 10^{19}$ & $398-434$ \\
$\mathrm{CdCl}_{2} . \mathrm{dmf} *$ & 176 & 0,50 & $2,54 \times 10^{19}$ & $425-440$ \\
$\mathrm{CdBr}_{2} \cdot \mathrm{dmf}$ & 101 & 0,17 & $1,07 \times 10^{9}$ & $387-458$ \\
\hline
\end{tabular}

* composto obtido pela degradação térmica do $\mathrm{CdCl}_{2} \cdot 2 \mathrm{dmf}$

Provavelmente, a etapa determinante na decomposição térmica desses compostos, seja a ruptura da ligação metal-ligante. Sendo assim, é de se esperar que exista algum tipo de relação numérica entre $E_{a}$ e $D(M-O)$ (entalpia de ligação metal-oxigênio). As entalpias de ligação metal-oxigênio para $\mathrm{CdCl}_{2} .2 \mathrm{dmf}, \mathrm{CdCl}_{2}$.dmf e $\mathrm{CdBr}_{2} \cdot \mathrm{dmf}$ são de 135,208 e $187 \mathrm{~kJ} \mathrm{~mol}^{-1}$ respectivamente ${ }^{4,5}$, mostrando que $\mathrm{E}_{\mathrm{a}}$ e $\mathrm{D}(\mathrm{M}-\mathrm{O})$ aumentam paralelamente.

$\mathrm{O}$ fato de $\mathrm{n}$ e $\mathrm{A}$ serem diferentes para $\mathrm{CdCl}_{2}$.dmf e $\mathrm{CdCl}_{2} \cdot \mathrm{dmf}^{*}$, indica que o modo de preparação do composto certamente é também fator determinante em sua cinética de decomposição, provavelmente devido a diferenças de natureza estrutural, seja a nível molecular ou em termos de forma e compactação dos grãos formados. Vale a pena perceber que, assim como n e A, a temperatura do início da degradação térmica $\mathrm{t}_{\mathrm{i}}$ também é maior para o $\mathrm{CdCl}_{2} \cdot \mathrm{dmf}^{*}$.

Comparando-se os valores de $\mathrm{A}$ para o $\mathrm{CdCl}_{2}$. dmf e o $\mathrm{CdBr}_{2} . \mathrm{dmf}$, constata-se uma diferença muito significativa, que não pode ser explicada simplesmente em função de possíveis diferenças estruturais, uma vez que os difratogramas de raios $\mathrm{X}$ (método do pó) sugerem serem estes compostos isomorfos ${ }^{5}$. A explicação pode talvez ser encontrada nas diferentes vias de síntese utilizadas para os dois compostos ${ }^{5,6}$, o que pode ter determinado diferenças nos tamanhos e formatos dos grãos produzidos, alterando assim sua área superficial, o que certamente influencia a cinética de decomposição. Assim, os valores de $\mathrm{A}$, ao menos para a cinética de termodecomposição de compostos de coordenação, parecem se relacionar com o que se poderia chamar de macroestrutura dos compostos, ou seja, as características morfológicas dos grãos formados.

\section{2) $\mathrm{CdCl}_{2.1,5} \mathrm{dmeu}$}

Pelo fato da curva DSC obtida não ser bem definida, optouse por efetuar o cálculo dos parâmetros cinéticos para este composto, utilizando-se os dados de termogravimetria ${ }^{8}$.

$\mathrm{O}$ composto degrada-se térmicamente em duas etapas:

$1^{\text {a })} \mathrm{CdCl}_{2} \cdot 1,5 \mathrm{dmeu}(\mathrm{s})=\mathrm{CdCl}_{2}$. dmeu (s) $+1 / 2 \mathrm{dmeu}(\mathrm{g})$

$\left.2^{\mathrm{a}}\right) \mathrm{CdCl}_{2}$.dmeu $(\mathrm{s})=\mathrm{CdCl}_{2}(\mathrm{~s})+$ dmeu $(\mathrm{g})$

Os cálculos foram efetuados utilizando-se programas em linguagem Basic, desenvolvidos por H. S. Júnior (Tabela 2).

Percebe-se que, de um modo geral, os valores de $E_{a}, n$ e A obtidos através dos cinco métodos utilizados, concordam entre si, dentro de um limite tolerável.

Os valores obtidos para a segunda etapa da degradação térmica, assemelham-se aos encontrados para os compostos com dmf que apresentam mesma estequiometria que o intermediário $\mathrm{CdCl}_{2}$. dmeu (que não chegou a ser sintetizado), indicando que a geometria dos compostos constitui-se em fator determinante para a cinética de decomposição. $\mathrm{O}$ fato dos valores de $\mathrm{E}_{\mathrm{a}}, \mathrm{n}$ e $\mathrm{A}$ serem bastante maiores para a primeira etapa que para a segunda, parece confirmar esta hipótese.

Em face dos elevados valores de A, deve-se esperar que a primeira etapa de decomposição seja mais rápida que a segunda, o que pode ser realmente constatado analisando-se a curva TG.

Novamente, elevados valores de A, parecem relacionar-se à morfologia dos grãos do composto, ficando implícito que, quanto mais finamente divididos forem os grãos (quanto maior a área superficial), maiores serão os valores de A.

Uma vez que a dmeu é um ligante monodentado, e não bi ou polidentado, a existência de uma estequiometria fracionária pode ser explicada como consequência da polimerização do composto. Uma vez que os valores de $n$ são de duas a três vezes maiores para a primeira etapa, a existência de um elevado valor para $\mathrm{n}$, parece associar-se de alguma forma à presença de uma geometria irregular para o composto, e não apenas à presença de um número maior de ligantes (veja-se por exemplo o valor de $n$ para o $\mathrm{CdCl}_{2}$. $2 \mathrm{dmf}$ ).

Tabela 2. Parâmetros cinéticos para o aduto $\mathrm{CdCl}_{2} \cdot 1,5 \mathrm{dmeu}$ $1^{\mathrm{a}}$ etapa

\begin{tabular}{cccccccc}
\hline método & $\mathrm{E}_{\mathrm{a}}(\mathrm{I})$ & $\mathrm{n}$ & $\mathrm{A}$ & correlação & $\mathrm{T}_{\mathrm{i}}-\mathrm{T}_{\mathrm{f}}(\mathrm{K})$ & $\alpha_{\mathrm{i}}-\alpha_{\mathrm{f}}$ & $\mathrm{B}$ \\
\hline $\mathrm{a}$ & 611,7 & 1,8 & $2,0 \times 10^{73}$ & 0,9935 & $394-405$ & $0,04-0,93$ \\
$\mathrm{~b}$ & 673,6 & 1,8 & $*$ & 0,9280 & $392-407$ & $0,007-0,99$ \\
$\mathrm{c}$ & 599,8 & 1,6 & $5,8 \times 10^{76}$ & 0,9985 & $394-406$ & $0,004-0,97$ \\
$\mathrm{~d}$ & 699,9 & 1,9 & $3,8 \times 10^{87}$ & 0,9879 & $392-406$ & $0,007-0,97$ \\
$\mathrm{e}$ & 571,3 & 1,7 & $1,26 \times 10^{74}$ & 0,9954 & $395-405$ & $0,007-0,93$ & 13 \\
\hline
\end{tabular}

$\mathrm{a}=$ Coats-Redfern, $\mathrm{b}=$ Freeman, $\mathrm{c}=$ Horovitz, $\mathrm{d}=$ Blazejowski, $\mathrm{e}=$ Zsakó

$\mathrm{I}=\mathrm{kJ} \mathrm{mol}^{-1}, \alpha=$ grau de avanço da reação, $\mathrm{B}=\mathrm{n}^{\mathrm{o}}$ de dados

* este método não permite calcular o valor do fator pré-exponencial

\begin{tabular}{cccccccc}
$2^{\mathrm{a}}$ etapa & \multicolumn{1}{c}{} \\
\hline método & $\mathrm{E}_{\mathrm{a}}(\mathrm{I})$ & $\mathrm{n}$ & $\mathrm{A}$ & correlação & $\mathrm{T}_{\mathrm{i}}-\mathrm{T}_{\mathrm{f}}(\mathrm{K})$ & $\alpha_{\mathrm{i}}-\alpha_{\mathrm{f}}$ & $\mathrm{B}$ \\
\hline $\mathrm{a}$ & 115,9 & 0,3 & $6,6 \times 10^{10}$ & 0,9980 & $455-511$ & $0,004-0,95$ \\
$\mathrm{~b}$ & 145,8 & 0,7 & $*$ & 0,9932 & $457-511$ & $0,004-0,95$ \\
$\mathrm{c}$ & 139,3 & 0,7 & $3,7 \times 10^{12}$ & 0,9988 & $450-512$ & $0,003-0,96$ \\
$\mathrm{~d}$ & 117,8 & 0,3 & $8,8 \times 10^{7}$ & 0,9974 & $450-512$ & $0,003-0,96$ \\
$\mathrm{e}$ & 114,8 & 0,3 & $1,3 \times 10^{9}$ & 0,9978 & $450-510$ & $0,003-0,94$ \\
\hline
\end{tabular}


Uma vez que os valores de $\mathrm{E}_{\mathrm{a}}$ são muito elevados para a primeira etapa, em relação a uma entalpia de ligação metaloxigênio relativamente baixa $\left(154,9 \mathrm{~kJ} \mathrm{~mol}^{-1}\right)^{8}$ para este composto, diferentemente dos compostos com dmf, grande parte do valor de $E_{a}$ parece estar comprometido com outros processos além da ruptura da ligação metal-ligante, como difusão do produto gasoso formado através do composto sólido remanescente, dessorção do produto gasoso formado da superfície do composto sólido remanescente, e rearranjos de natureza estrutural, quer da micro ou da macro estrutura do composto sólido.

\section{REFERÊNCIAS}

1. Lagowski, J. J.; The Chemistry of Nonaqueous Solvents; vol. IV, Academic Press, 1976.

2. Sakurai,H.; Kondo, F.; J. Org. Chem. 1976, 117, 149.

3. Rosenfarb, J.; Caruzo, J. A.; Can. J. Chem. 1976, 54, 3492.
4. Brown, M. E.; Phillpotts, C. A. R.; J. Chem. Educ. 1978, $55,556$.

5. Oliveira, O. A. de; Tese de Doutorado; IQ - Unicamp, 1981.

6. Oliveira, O. A. de; Airoldi,C.; Chagas, A.P.; Inorg. Chem. 1983, 22, 136.

7. Rogers, R. N.; Smith, L. C.; Thermochim. Acta 1970, 1,1.

8. Farias, R. F.de; Oliveira, O. A. de; Quím. Nova 1996, $19,100$.

9. Coats, A. W.; Redfern, A. P.; Nature 1964, 68, 201.

10. Freeman, S.; Caroll, B.; J. Phys. Chem. 1958, 62, 394.

11. Horowitz, H. H.; Metzger, G.; Anal. Chem. 1963 , 35,1464

12. Blazejowski, I; Thermochim. Acta 1984, 76, 359.

13. Zsako, J.; J. Phys. Chem. 1968, 72, 2406.

14. Harris, D. C.; Quantitative Chemical Analysis; W. H. Freeman and Company, New York, 1995. 